\title{
Juristen und Sprachkultur
}

\section{Lawyers and Language Culture}

\section{Karsten Rinas}

\begin{abstract}
This article discusses the influence of lawyers on language culture. First, jurisprudence is identified as a specific form of philology, which explains its affinity to questions of language culture. This is followed by a sketch of the lawyers' influence on German language culture which is based on historical examples. Finally, the article offers a critical discussion of the official bureaucratic use of German language („Kanzleistil”) which was mainly founded by lawyers.
\end{abstract}

\section{Keywords}

language culture, lawyers, history of language, grammar, spelling, official bureaucratic German language ( ${ }_{\text {"Kanzleistil") }}$ 


\section{Einleitung}

Wer sich mit historischen sprachkulturellen - etwa grammatischen, (normativ-)stilistischen oder literarischen - Beiträgen im europäischen Raum befasst, wird kaum übersehen können, dass in diesen Bereichen die Juristen eine auffallend starke, mitunter sogar dominierende, Autorengruppe darstellen. Dies zeigt sich gerade auch in der Geschichte der deutschen Sprachkultur. Im Folgenden sollen in exemplarischer Form diverse solcher Beiträge aufgeführt werden, wobei historische Beiträge zur deutschen Interpunktionslehre besondere Berücksichtigung finden. ${ }^{1}$ Es werden aber auch andere Sachbereiche sowie andere Sprachkulturen thematisiert.

Im ersten Schritt soll den historischen und sachlichen Grundlagen für das Interesse der Juristen an sprachkulturellen Fragen nachgegangen werden.

\section{Grundlagen}

\subsection{Jurisprudenz und Philologie}

Im Jahre 1837 gab es in Paris einen bemerkenswerten Zweikampf, von dem seither sporadisch in anekdotischer Form berichtet wird: ${ }^{2}$ Zwei Professoren der Pariser juristischen Fakultät waren unterschiedlicher Auffassung darüber, wie eine bestimmte Stelle in JUSTINIANs Pandekten in einer modernen Edition zu interpungieren sei; der eine plädierte für den Gebrauch des Kolons, der andere wollte hier das Semikolon setzen. Hierüber gerieten die beiden Professoren dermaßen in Streit, dass es zu einem Duell kam, bei welchem der Anhänger des Semikolons am Arm verletzt wurde.

Man kann diese Anekdote als bizarres Beispiel blinden Gelehrteneifers deuten - und als solche wird sie auch gewöhnlich präsentiert. Sie wirft jedoch auch ein bezeichnendes Schlaglicht darauf, dass gerade die sprachliche Gestaltung für Juristen von enormer Bedeutung ist. Und dies ist kein Zufall, sondern liegt in der Natur der Sache: Die Tätigkeit der Juristen ist stark philologisch geprägt, denn es geht hierbei wesentlich darum, Gesetze möglichst exakt zu fixieren bzw. vorhandene Gesetze detailliert und differenziert zu interpretieren. ${ }^{3}$ Diese Orientierung an Texten hat eine uralte Tradition, die letztlich bis in die Frühgeschichte reicht, denn es spricht einiges dafür, dass gerade die Erfordernisse der Gesetzgebung und Verwaltung in den frühen Hochkulturen ein wesentlicher Faktor bei der Entwicklung der Schrift waren. ${ }^{4}$ Und dies erklärt auch den enormen Stellenwert, den die Juristen der sorgfältigen Textgestaltung beimessen. Infolgedessen wurden

1 Dieser Beitrag basiert auf einer umfangreicheren Studie zur Geschichte der deutschen Interpunktionslehre (RINAS 2017).

2 Vgl. etwa schon The Family Magazine. Vol. IV 1837, S. 153. Vgl. auch JEANNENEY (2004: 57).

3 Zur Textgebundenheit im abendländischen Rechtswesen und deren Auswirkung auf unser Rechtsdenken vgl. WEITZEL (1994). Vgl. auch LERCH \& CHRISTENSEN (2016). Die Beziehungen der Jurisprudenz zu Rhetorik, Logik, Dialektik und Hermeneutik diskutiert KRAWIETZ (1996).

4 Vgl. etwa KIENAST (1969:45), KLIX (1993: 250-252). 
gerade bei der schriftlichen Wiedergabe von Gesetzen bereits früh die Möglichkeiten der Textgliederung und -gestaltung genutzt. Dies hat RAIBLE (1991:6-8) am Beispiel des Stadtgesetzes von Málaga aus dem 1. Jh. n. Chr. demonstriert, welches bereits ein erstaunlich modernes ,Layout‘ (Überschriften, Nummerierungen, Absatzgliederung) aufweist, obwohl diese Mittel damals keineswegs allgemein verbreitet waren.

\subsection{Jurisprudenz und Rhetorik}

Neben dieser notwendigen philologischen Orientierung gibt es eine weitere Verbindung von Recht und Sprachkultur, die bereits in der Antike geknüpft wurde: Eine wesentliche Motivation für die Entwicklung und Ausbreitung der Rhetorik lag zunächst in dem Umstand, dass die Redefähigkeit in den demokratischen griechischen Stadtstaaten besondere Bedeutung erlangt hatte. ${ }^{5}$ Es gab aber nicht nur öffentliche Debatten, sondern auch öffentliche Prozesse. Auch die hierbei zentralen Elemente - v.a. die Beweisführung - wurden in der Rhetorik eingehender reflektiert, sodass man mit einigem Recht von einer ,forensischen Rhetorik ${ }^{\prime 6}$ sprechen kann, die schon bei ARISTOTELES in Form der Gerichtsrede berücksichtigt wurde und später bei CICERO eine wichtige Rolle spielen sollte. Auch in der Folgezeit wurde die rhetorische Tradition gerade in den Rechtswissenschaften gepflegt. ${ }^{7}$

Wir können somit konstatieren, dass die Rechtswissenschaften bereits in der griechisch-römischen Antike eine ausgeprägte Affinität zu sprachkulturellen Fragen aufwiesen.

\section{Jurisprudenz und Sprachkultur im deutschen Sprachraum: Einige Streiflichter}

Die schon in der Antike ausgeprägte philologische Ausrichtung der Rechtswissenschaft setzte in nahezu ganz Europa spätestens mit der Rezeption des Corpus iuris civilis ein; in Deutschland begann diese Rezeption in der Mitte des 15. Jahrhunderts. ${ }^{8}$ Im Laufe des 15. und 16. Jahrhunderts, konnten sich die Juristen in Deutschland als Berufsstand etablieren, etwa als Richter oder Anwälte. ${ }^{9}$ Ein weiterer möglicher - und angesehener Beruf für Juristen war der des ,Stadtschreibers', d.h. des Leiters einer städtischen Kanzlei; solche Stadtschreiber waren praktisch die obersten Verwaltungsbeamten der Städte

5 Vgl. etwa STÖRIG (1988: 144-148), GÖTTERT (2009: 75-78).

6 Diesen Titel trägt das CICERO gewidmete Kapitel (C.2) in GÖTTERT (2009).

7 Vgl. auch ROCKINGER (1863:XVII-XVIII). Ein gutes Beispiel bietet auch das von ANONYMUS (1744)

skizzierte juristische Studienprogramm.

8 Vgl. SCHMIDT-WIEGAND (1998: 87f.).

9 Vgl. SCHMIDT-WIEGAND (1998: 88). 
und besaßen einen erheblichen Einfluss. ${ }^{10}$ Diesen Beruf übte z.B. der ,Dichterjurist Sebastian BRANT aus. ${ }^{11}$

In diesem Zeitraum wird im deutschen Sprachraum auch der sprachkultivierende Einfluss der Juristen greifbar, und zwar insbesondere in den sogenannten Formelbüchern (oder ,deutschen Rhetoriken'), welche seit dem ausgehenden 15. Jahrhundert in wachsendem Maße gedruckt wurden. ${ }^{12}$ Die Hauptfunktion dieser Werke bestand darin, Anleitungen und Muster für den amtlichen Briefverkehr und die Erstellung von Urkunden zu liefern; in diesem Zusammenhang wurden auch Fragen der Textgestaltung sowie diverse sprachliche Phänomene angesprochen. ${ }^{13}$

Die eigentliche Grammatikschreibung des Deutschen setzt erst in den 1570er Jahren ein, mit den Werken von Laurentius ALBERTUS (1573), Albert ÖLINGER (1573) und Johannes CLAJUS (1578). Hingegen behandeln die Formelbücher schon deutlich früher sprachliche Probleme des Deutschen, weshalb diese Werke in der Sprach- und Grammatikgeschichte auf Interesse gestoßen sind. Dies gilt zum Beispiel für den Schryfftspiegel $(1527)^{14}$. Besonders interessant ist das Werk Spiegel der waren Rhetoric (1493) des Kanzlisten und späteren Buchdruckers Friedrich RIEDERER. ${ }^{15}$ Auch dieses Buch bietet Anleitungen für den amtlichen Briefverkehr, geht jedoch auch deutlich darüber hinaus; es kann als die erste umfassende Darstellung der Rhetorik in deutscher Sprache angesehen werden. ${ }^{16}$ Diese Darstellung ist insgesamt stark den (klassischen) lateinischen Vorbildern verpflichtet, doch finden sich auch bereits Ausführungen, die auf speziellere Erscheinungen des Deutschen Bezug nehmen. So erläutert RIEDERER, dass das Hilfsverb haben sich auf mehrere Partizipien (bei RIEDERER ,Partickel') beziehen kann, ${ }^{17}$ was er u.a. an folgendem Beispiel illustriert:

(1) Herr Richter ich clag von Petern · nachdem ich im vor iaren ein guldin iärlich verzinset. vnd doch inn erbetten hab das er mich des zinses gütlich erlassen: ... (1493[2009]:125)

Hieran anschließend kritisiert RIEDERER das generelle Weglassen solcher Hilfsverben:

10 Vgl. HERGENHAHN (1995).

11 Vgl. SCHMIDT-WIEGAND (1998: 88f.).

12 Vgl. NICKISCH (1969:17-25, 248-260). Diese Bücher knüpften aber teils an die schon seit dem Hohen Mittelalter verbreiteten lateinischen Briefsteller und Formelbücher (ars dictandi) an, welche das Verfassen von Briefen und Urkunden lehrten. Auch hier gab es bereits eine starke Affinität zum Verwaltungs- und Rechtswesen; vgl. ROCKINGER (1863).

13 Im Übrigen brachten viele Juristen im 16. Jahrhundert der humanistischen Strömung ein starkes Interesse entgegen; vgl. HAMMERSTEIN (Hg.) (1996: 241f.).

14 Vgl. GÖTZ (1992), MOULIN-FANKHÄNEL (1994: 163-165).

15 Zu RIEDERER vgl. KNAPE \& LUPPOLD (2010: 12-15).

16 Vgl. KNAPE (2000: 207) sowie KNAPE \& LUPPOLD (2010).

17 Hier folgt RIEDERER allerdings lateinischen Vorbildern, namentlich der Grammatik von PUBLICIUS (1482); vgl. RIEDERER (1493 [2009]:125, Fn. 392). Aber RIEDERER ist der erste, der dies aufs Deutsche anwendet. 
„Aber etlich vnbericht $\cdot$ die doch meynend ettwas wissen vnd solichs von andern hörent oder in schrifften sehend: vnd doch den grund nit merckend: missbruchend dise zierd: damit . wenn sy meynend subtyl vnd clůg sin: vermydend sy die besliessenden wort in allen partickeln der red / eben zum letsten als in den andern: das gebürt ein vnuerstentlichen sinn“. (1493 [2009]: 126)

Sein Negativ-Beispiel hierzu lautet (ebd.):

(2) Nachdem ir mir früntschaft bewyset / ich nit vm üch verdient / aber bereit / vnd darumb ietz zů üch komen / solichs zůuerdienen.

Hier gilt:

„Diewort .haben. vnnd .bin. stond nit in besluß yeder partickel / dahin sich dann wolgebürt Deßhalb ist solichs mer entschöpffung denn gezierd der red: Darumb das sy gantz heruß blyben." (ebd.)

Des Weiteren gibt RIEDERER den Hinweis, dass bei Aufzählungen vor dem letzten Glied „vnd“ eingefügt werden soll (ebd.).

Ausführungen zu derart komplexen Konstruktionen sollten in der Grammatikschreibung noch längere Zeit auf sich warten lassen. RIEDERERs diesbezügliche Darlegungen können als Pionierleistung für das Deutsche angesehen werden. Und mit seiner Verurteilung der Hilfsverben-Tilgung schlägt er ein Thema an, das in der deutschen Sprachpflege noch die nächsten 500 Jahre diskutiert werden sollte. ${ }^{18}$

Auch im 17. Jahrhundert beteiligen sich die Juristen rege an der deutschen Sprachkultur. ${ }^{19}$ Dies gilt für die ,Dichterjuristen“ Friedrich von LOGAU und Andreas GRYPHIUS, es gilt auch für den Dichter und Dichtungstheoretiker Martin OPITZ (Buch von der Deutschen Poeterey, 1624), und es gilt ebenso für den herausragenden Sprachgelehrten Justus Georg SCHOTTEL, der mit seinem Werk Ausführliche Arbeit von der Teutschen HaubtSprache (1663) die damals umfassendste und innovativste Darlegung zur deutschen Sprache vorlegte. ${ }^{20}$ Genannt werden können auch noch Kaspar VON STIELER und Georg Philipp HARSDÖRFFER, die als Verfasser einflussreicher Briefsteller, aber auch anderer verdienstvoller Beiträge hervorgetreten sind, STIELER vor allem mit seinem voluminösen Wörterbuch Der teutschen Sprache Stammbaum und Fortwachs (1691), dem eine Kurze Lehrschrift Von der Hochteutschen Sprachkunst angehängt ist, in welcher erstmals differenzierte Ausführungen zur Wortstellung gegeben werden. ${ }^{21}$ Und HARSDÖRFFER

18 Im heutigen Deutsch ist diese Erscheinung praktisch ausgestorben. Eine Verurteilung dieser Konstruktion findet sich aber etwa noch bei von WOLZOGEN (1890: 45 f.) oder WUSTMANN (1903: 134-139). Und sogar noch ENGEL (1931: 73 f.) und REINERS (1961: 342 f.) diskutieren dieses Phänomen (wenn auch auf differenziertere Weise). Vgl. auch RINAS (2011: 19f., 164).

19 Vgl. SCHMIDT-WIEGAND (1998: 90f.).

20 Vgl. etwa EGGERS (1986: 199), GARDT (1999: 119).

21 Vgl. JELLINEK (1914: 372f.). 
hat sich u.a. mit der Poetik auseinandergesetzt und hierbei auch Fragen der sprachlichen Korrektheit angesprochen. So bietet er etwa in seinem Werk Poetischer Trichter (1647) eine ungewöhnlich komplexe Interpunktionslehre (S. 118-123). ${ }^{22}$

Die sprachkulturellen Aktivitäten dieser Autoren hingen eng mit ihrer beruflichen Tätigkeit als ,Sekretäre‘ bzw. Beamte zusammen: „Anteil an der Sprachpflege zu haben, gehörte zu ihrem Prestige.“23 In dieser Zeit wirkten viele Juristen auch in den sprachpflegerisch engagierten Sprachgesellschaften ${ }^{24}$ mit: „Der Anteil der Juristen“ in diesen Gesellschaften ist „nicht zu übersehen, wenn auch weder von Rechtshistorikern noch von Philologen auf ihre Wirkung hin untersucht“".25

Ein interessantes Beispiel für die enge Verknüpfung von Rechtspflege und Sprachkultur bietet die Abhandlung Send=Schreiben/ An Einen Vornehmen Freund... Betreffend die Doctrinam Notarum Distinctionum, E Interpunctionum (1687) des Juristen Johannes Hieronimus IMHOF (auch IMHOF/IMHOFF), welche man als erste deutschsprachige monographische Darstellung zur Interpunktion ansehen könnte. ${ }^{26}$ Hierbei wird u.a. die Interpunktion als notwendiges Mittel für das Textverständnis ausgewiesen, indem strittige Interpunktionsfälle aus Theologie und Politik als Negativbeispiele diskutiert werden (S. 6-12).

Das Interesse der Juristen an sprachlichen Fragen riss auch im 18. Jahrhundert nicht ab. Der Jurist und Hofbeamte Friederich Carl von MOSER ${ }^{27}$ legte 1749 gar den Versuch Einer Staats-Grammatic vor, in welchem er das Ziel verfolgte, „die Wichtigkeit der grammaticalischen Regeln in Staats-Sachen und deren verschiedene Abweichungen von der Sprache der grossen Welt zu zeigen, die besondere Fälle, wo über den Verstand und Bedeutung der Wörter oder sonst aus einer grammaticalischen Veranlassung zwischen grossen Herren Streitigkeiten entstanden, zu sammlen“ (Vorrede). Hierbei diskutierte er auch - ähnlich wie vorher IM-HOF - einige interessante Probleme, die durch einen unklaren Interpunktionsgebrauch entstanden (S. 28-34).

Als weiteres Beispiel kann der Staatsrechtler Johann Stephan PÜTTER genannt werden, welcher seiner weit verbreiteten Anleitung zur Juristischen Praxi einige Zugaben folgen ließ, die „insonderheit von der Orthographie und Richtigkeit der Sprache“ handeln (1767, „Zweyte Zugabe“ (zum §.22); S. 11-50). Dies rechtfertigt er folgendermaßen:

„Regeln der Teutschen Sprache und Orthographie sollten zwar billig nicht erst in der Anleitung zur juristischen Praxi ihren Platz finden, sondern längst vorher als bekannt vorausgesetzt werden. Allein der offenbare Mangel, der sich hierinn zeiget, und der daraus entstehende Uebelstand, der einen Rechtsgelehrten mehr, als andere, verunzieret, machen es zur Nothwendigkeit, gewisse Hauptregeln auch bey dieser Gelegenheit näher einzuschärfen.“ (S. 11)

22 Vgl. hierzu ausführlicher RINAS (2017: 108-113).

23 SCHMIDT-WIEGAND (1998: 91).

$24 \mathrm{Zu}$ den barocken Sprachgesellschaften vgl. etwa VON POLENZ (1994: 112-119)/(2000).

25 SCHMIDT-WIEGAND (1998: 90).

26 Allerdings ist dem einschränkend hinzuzufügen, dass diese Arbeit nur 24 Seiten umfasst, zudem in einer deutsch-lateinischen Mischsprache geschrieben ist und vorwiegend lateinische Beispiele anführt. Zu diesem Werk vgl. auch RINAS (2017: 115f.).

27 Zu MOSER vgl. etwa GLÜCK (2013: 47-50). 
„Da die juristische Praxis durch nichts anders als durch mündliche Vorträge oder schriftliche Aufsätze zur Ausübung kömmt; so ist ein Teutscher Rechtsgelehrter in seinem ganzen Berufe mit der Sprache beschäfftiget, und zwar unter dem Angesichte grosser Herren, oder hoher Staats-Bedienten, Landes- und Justitz Collegien, oder anderer Obrigkeiten, jedesmahl bey viel zu ernsthaften Gelegenheiten, als daß es gleichgültig seyn könnte, unachtsam und fehlerhaft oder richtig und regelmäßig mit der Sprache und Schreibart umzugehen.“ (ebd.)

Die Liste sprachkulturell engagierter Rechtswissenschaftler ließe sich mühelos fortsetzen. Auch Jacob GRIMM war bekanntlich studierter Jurist und wurde auf dem ,Umweg der Rechtsgeschichte zum Studium der Sprachgeschichte inspiriert. ${ }^{28}$ Gerade für die Frühphase der Institutionalisierung der deutschen Philologie an der Wende zum 19. Jahrhundert gilt, dass die germanistisch-philologischen Tätigkeiten oft ,im Nebenberuf von Hochschullehrern ausgeübt wurden, die primär einer anderen Fachrichtung zugehörten. Dies gilt etwa für zwei im philologischen Bereich äußerst produktive Staatsrechtler, Karl Heinrich Ludwig PÖLITZ und Friedrich Jakob SCHMITTHENNER. ${ }^{29}$ PÖLITZ ist u.a. mit voluminösen Beiträgen zur deutschen Sprachkunde und Stilistik hervorgetreten $(1804,1805)$; SCHMITTHENNER publizierte u.a. die umfangreiche deutsche Sprachlehre Teutonia (1828). Beide Autoren legten u.a. auch wichtige Beiträge zur Interpunktionslehre vor. ${ }^{30}$ Als weiterer einschlägiger Beitrag ließe sich die von dem Juristen und nachmaligen Verlagsdirektor Alexander ELSTER 1901 vorgelegte geistreiche Studie zur Interpunktionstheorie nennen. ${ }^{31}$

Es fügt sich ins Bild, dass am Ende des 19. Jahrhunderts, als sich die Bemühungen um eine vereinheitlichte deutsche Rechtschreibung intensivierten, auch der Gedanke diskutiert wurde, das Bürgerliche Gesetzbuch zur Grundlage dieser Vereinheitlichung zu machen. ${ }^{32}$ Zudem gab es in dieser Zeit noch eine interessante Diskussion über den Fremdwortgebrauch im BGB, die im Beitrag von ERLER (1896) reflektiert wird. ${ }^{33}$

Schließlich sei noch erwähnt, dass auch zwei einflussreiche Sprachkritiker des 20. Jahrhunderts eine juristische Bildung besaßen: Sowohl der deutschböhmische Theaterkritiker, Philosoph und Sprachkritiker Fritz MAUTHNER als auch der österreichische Schriftsteller und Sprachkritiker Karl KRAUS studierten die Rechtswissenschaft, MAUTHNER in Prag, KRAUS in Wien. ${ }^{34}$ Allerdings haben beide Autoren dieses Studium nicht beendet. Dennoch darf gemutmaßt werden, dass ihr Interesse an subtilen Sprachfragen auch durch ihre juristischen Kenntnisse forciert wurde. ${ }^{35}$

28 Vgl. hierzu SCHMIDT-WIEGAND (1987). Allerdings ist GRIMMs Beitrag zur Sprachkultur durchaus ambivalent; vgl. hierzu RINAS (2011: 26-29).

29 Vgl. BAHNER \& NEUMANN (Hgg.) (1985: 206f.).

30 Vgl. RINAS (2017: 218-224).

31 Vgl. RINAS (2017: 283-285).

32 Vgl. etwa NERIUS (Hg.) (2007: 347).

33 Vgl. SCHMIDT-WIEGAND (1998: 94).

34 Zu KRAUS vgl. etwa IGGERS (1967: 1), zu MAUTHNER vgl. KÜHN (1975: 111-115).

35 Die Widerspiegelung juristischer Probleme und Denkweisen im literarischen Werk von KRAUS diskutiert MERKEL (1998). 


\section{Das schwierige Erbe: Der Kanzleistil}

Die bislang genannten Beispiele dürften zur Genüge illustrieren, dass von juristischer Seite wertvolle Impulse für die deutsche Sprachkultur ausgingen. Allerdings war dieser Einfluss der Juristen durchaus ambivalent, denn es gab auch problematische Erscheinungen. Hierzu gehört insbesondere der viel gescholtene Kanzleistil. Dessen Geschichte und Rezeption sollen in diesem Abschnitt skizziert werden.

Seit dem ausgehenden 15. Jahrhundert gab es im deutschen Sprachraum Bemühungen, Wissensbestände in deutschsprachigen Büchern zu reflektieren und damit auch eine deutsche Fachsprache zu etablieren. Besonders deutlich manifestierte sich dies an der Wende zum 16. Jahrhundert, als „eine ganze Reihe herausragender, für ihr Diskursfeld neue Maßstäbe setzender Werke" erschien (KNAPE \& LUPPOLD 2010: 11). Genannt werden können hier neben dem in Abschnitt 3 behandelten Spiegel der waren Rhetoric (1493) von Friedrich RIEDERER für den literarischen Bereich Sebastian BRANTs Narrenschiff (1494), für den juristischen Ulrich TENNGLERs Rechtskompendium Layen Spiegel (1509) und für den theologischen Bereich LUTHERs Übersetzung des Neuen Testaments (1522). Als weiteres ,Sachbuch' ließe sich etwa die erste deutschsprachige Logik von Ortolf FUCHSBERGER (1533) anführen. ${ }^{36}$ - Diese Entwicklung ließe sich auch zum Entstehen eines ,deutschen Nationalbewusstseins' in dieser Epoche in Beziehung setzen. ${ }^{37}$

Dass in dieser Phase das Interesse an den Volkssprachen zunahm, bedeutet allerdings nicht, dass die Gelehrten dieser Zeit dazu übergingen, ,dem Volk aufs Maul zu schauen', also dessen Ausdrucksweisen direkt zu übernehmen. Eine derart enge Anbindung an die Alltagssprache wurde nicht angestrebt - nicht einmal von LUTHER. Vielmehr wurde auch beim ,volkssprachlichen' Gebrauch ein aus der antiken Rhetorik ererbtes Prinzip reflektiert: das Prinzip der ,Angemessenheit ${ }^{\circ}$ (aptum) ${ }^{38}$ Diesem schon von den rhetorischen Klassikern (ARISTOTELES, CICERO, QUINTILIAN) hervorgehobenen Grundsatz zufolge muss man für sprachliche Äußerungen die situativ angemessene Form bzw. Ausdrucksweise wählen. Dieser Grundsatz wurde in der Frühen Neuzeit nicht nur übernommen, sondern im Kontext der Ständegesellschaft sogar noch verschärft. ${ }^{39}$ Wie diese frühneuzeitliche Ständegesellschaft funktionierte, hat VAN DÜLMEN (1981) beschrieben: ${ }^{40}$ Es war eine Gesellschaft, ,in der jeder einzelne durch Geburt oder Privileg Mitglied eines Standes war und aufgrund dieser Zugehörigkeit Anspruch auf die von seinem Stand monopolisierten Lebenschancen besaß.“ Es gab somit „eine rigid festgeschriebene und erstmals auch herrschaftlich abgesicherte Ständeordnung“ mit „einer fast starren Ständestruktur“, „in der jede Gruppe und jeder einzelne erstmals eine klar definierte Rolle zugewiesen bekam, der er sich bei Verlust von Ehre und Privi-

36 Vgl. hierzu BRITZELMAYR (1947).

37 Vgl. hierzu GIESECKE (1992: 108-110) sowie die Abhandlung von LAU (2010).

38 Vgl. hierzu den historischen Überblick von ASMUTH (1992).

39 Vgl. ASMUTH (1992: 593).

40 Vgl. etwa auch CHAUNU (1968: 461-500), HINRICHS (1980: 66-80), KESSEL (2008: 44f.). 
leg fügen mußte“. Dies führte zu „einer tendenziell alle Bereiche des Alltagsverhaltens normativ regelnden, konventionell geprägten standesgemäßen Lebensführung, die die soziale Vorrangstellung des Adels und die Untertänigkeit des Volkes unter der politischen Führung eines Fürsten garantierte" (S. 20f.). - In einem solchen Kontext sind beispielsweise die Kleidung und die Umgangsformen ebenso ein Statussymbol wie der Sprachgebrauch. Auch der Gebrauch des Deutschen war hiervon nicht ausgenommen, insbesondere dann nicht, wenn es um schriftliche Äußerungen ging. Dem Schriftgebrauch haftete generell noch ein besonderer Nimbus an, weil er in der Frühen Neuzeit noch keineswegs selbstverständlich war. Prototypisch für den Schriftgebrauch waren akademische, theologische und juristische Kontexte - allesamt Situationen, in denen sozial höher stehende Personen sich über ,bedeutende‘ Angelegenheiten austauschen, was einen ,gehobenen' Sprachgebrauch forcierte. ${ }^{41}$ Namentlich eine komplex gestaltete Sprache drückt dieses Moment geradezu ikonisch aus, denn hierbei wird das „Prinzip der Analogie zwischen sprachlichem (aktionalem) Mehraufwand und sozial gehobener Stellung“ reflektiert (SCHWITALLA 2002: 392). ${ }^{42}$ Und dies zeigte sich besonders deutlich in der Verwaltungssprache, der sogenannten ,Kanzleisprache‘: 43 „Von Anfang an trug die Kanzleisyntax die konnotative Aura von Macht und Herrschaft mit sich“ (SCHWITALLA 2002: 379).

Dieses Bemühen um eine adäquate komplexe Ausdrucksweise hat der Kanzleisprache schon früh einen schlechten Ruf eingebracht und sie zum Gegenstand der Kritik und des Spottes werden lassen. Beispiele hierfür finden sich spätestens im 16. Jahrhundert. ${ }^{44}$ Und selbst der Germanist Arno SCHIROKAUER (1952: 1018f.) tradiert noch diese Kritik, wenn er formuliert, dass die frühneuhochdeutschen Texte „der Buchhalter und Notare“

„auf einem mit Totem-Zeichen abgesteckten Feld wuchern, auf dem das sprachliche Leben stockt; ihre traditions-geheiligten Formeln sind vom Sprachleben ausgeschlossen, nur im Bereich des Gerichts existiert, was sie umständlich und gespreizt ,tun kund und zu wissen‘. Es ist schon wahr, daß den Fluten von Kanzleipapier damals niemand entgehen konnte, daß zahllose Briefsteller und Formularien, Sammlungen von Musterbriefen und Spiegel der wahren Rhetorik dem um ein höheres Deutsch Verlegenen nachstellten, aber doch nur dann, wenn er in schriftlichen Austausch mit seinen Behörden zu treten wünschte. Es war ,Deutsch mit einem Kratzfuß‘, feiertäglich, ungewohnt, eine Audienzsprache zu gewissen Gelegenheiten respektvollst anzulegen wie ein Galanterie-Degen.“

Insofern man diese Ausführungen als Beschreibung dessen liest, wie diese Sprachvarietät auf einen heutigen Sprachbenutzer wirkt, wird man ihnen eine gewisse Berechtigung nicht absprechen können. Aus genuin philologischer Sicht ist SCHIROKAUERs

41 Vgl. auch die in Abschnitt 3 zitierten Ausführungen von PÜTTER (1767: 11).

42 Zum Verhältnis von Repräsentationsstil und Ständegesellschaft vgl. etwa auch SCHWIND (1977: 29-34).

43 Vgl. hierzu auch KNAPE \& LUPPOLD (2008). Vgl. etwa auch BENTZINGER (2000). Ein umfassendes Kompendium zur Kanzleisprache ist GREULE/MEIER/ZIEGLER (Hgg.) (2012).

44 Vgl. SCHWITALLA (2002: 387-391). 
Kritik hingegen problematisch. Aufgabe einer philologisch-historischen Betrachtung sollte sein, die uns fremden Konventionen aus ihrem historischen Kontext heraus verständlich zu machen (was natürlich nicht bedeutet, dass man sie verteidigen müsste). ${ }^{45}$ Vor dem Hintergrund der Ständegesellschaft lässt sich auch das ,pompöse' Sprachverhalten als Widerspiegelung der sozialen Ordnung bzw. des sozialen Ranges deuten. ${ }^{46}$

Wir können somit konstatieren, dass im Humanismus ein komplexer und ,erhabener Sprachgebrauch zumindest in bestimmten Kontexten ein erstrebenswertes Ideal war.

Der hier beschriebene ,erhabene' Sprachgebrauch blieb nicht auf juristische Kontexte beschränkt, sondern wirkte sich auch in der Literatur aus, etwa in der manieristischen ,Zweiten Schlesischen Schule', vor allem bei Daniel Caspar VON LOHENSTEIN und Christian HOFFMANN VON HOFFMANSWALDAU. Erst im Laufe des 18. Jahrhunderts kam es zu einem markanten Wandel: In dieser Zeit wurde der repräsentative, an die Ständegesellschaft gebundene Kanzleistil allmählich durch einen schlichteren, der Alltagssprache näher stehenden Stil verdrängt. In wachsendem Maße wurde dieser auf Erhöhung und Repräsentation bedachte Stil mit der negativ konnotierten Bezeichnung ,Schwulst' belegt. Auch im literarischen Bereich wurde der Schwulst-Stil kritisiert. ${ }^{47}$

Eine deutliche Abgrenzung zeigt sich bei den französischen Vorbildern folgenden ,galanten' Autoren, für deren Schreiben gerade konstitutiv ist, dass es nicht ,bombastisch“ sein soll. Diese Entwicklung setzte bereits im ausgehenden 17. Jahrhundert ein, wobei als frühe Vertreter Christian THOMASIUS und Christian WEISE genannt werden können. Hatte man zu SCHOTTELs Zeiten „zumeist nur auf den Wortschatz gesehen, um ihn von allem Fremden zu befreien und durch Neubildungen zu ergänzen, so wollten Thomasius, Christian Weise und ihre Nachfolger den deutschen Sprachstil im ganzen verfeinern. ,Stilbildung“ wurde das Leitwort der deutschen Spracherziehung im 18. Jahrhundert" (FRANK 1976: 81). Dies wird etwa in Christian WEISEs rhetorischen Schriften reflektiert, wo bereits ein gemäßigter Einsatz sprachschmückender Mittel empfohlen wird. ${ }^{48}$ (Später wurde WEISE gar als LOHENSTEINs Antipode und als Gegner der Zweiten Schlesischen Schule gesehen. ${ }^{49}$ ) Andere ,galante‘ Autoren plädieren noch deutlicher für den Gebrauch übersichtlicher, nicht ausufernder Perioden, etwa August BOHSE (= TALANDER) und Christian Friedrich HUNOLD (= MENANTES). ${ }^{50}$ Wesentlich schärfere Kritik am ,Schwulst' findet sich dann bei GOTTSCHED und GELLERT. ${ }^{51}$ Allerdings gilt auch für diese Kritiker die Einsicht, dass die Revolution ihre Kinder frisst:

45 Dies ist auch das erklärte Ziel der historischen Anthropologie bzw. der Mentalitätsgeschichte. Vgl. hierzu etwa DRESSEL (1996: 180-184) und DINZELBACHER (Hg.) (2008: XXXVII-XL).

46 Vgl. etwa auch KNAPE \& LUPPOLD (2010: 121f.).

47 Eine grundlegende Studie zum literarischen Schwulst ist SCHWIND (1977). Vgl. auch ZYMNER (2007).

48 Vgl. etwa SCHWITALLA (2002: 189f.), SCHWIND (1977: 153-169), NICKISCH (1991: 79f.).

49 Vgl. BARNER (1984: 691). Diesen Gegensatz hob laut WENDLAND (1930: 137) bereits der WEISESchüler Andreas KÖHLER hervor.

50 Vgl. SCHWITALLA (2002: 189f.).

51 Vgl. SCHWITALLA (2002: 190f.); zu GELLERT vgl. etwa NICKISCH (1991: 81f.), zu GOTTSCHED vgl. SCHWIND (1977: 205-257). Sehr dezidiert hat diese Kritik auch LESSING vorgetragen; vgl. etwa VELLUSIG (2000: 117). 
Späteren Kritikern galten auch Autoren wie WEISE, BOHSE und GOTTSCHED als Exponaten eines ,Schwulst-Stils‘. Er wurde schließlich der gesamten Barockzeit attestiert. ${ }^{52}$

Besonders deutlich manifestierte sich dieser Bruch zunächst in der Briefkultur. Dies hing zusammen mit dem im 18. Jahrhundert rapide zunehmenden Briefverkehr, der ,Briefwut ${ }^{6}{ }^{53}$ Die Briefschreiber waren nun nicht nur Personen, „die mit dem Schreiben von Briefen ein professionelles Interesse verbinden (also Sekretäre, Juristen, Gelehrte), sondern auch solche, die nicht in eine von Latinität und Rhetorik geprägte akademische Schriftkultur sozialisiert wurden (und das waren bekanntlich v.a. die Frauen)" (VELLUSIG 2011: 158). ${ }^{54}$ Und man schrieb nunmehr „nicht nur solche Briefe, die in den alten Briefstellern rubriziert sind, sondern auch solche, die es bislang noch nicht gab, weil sie sich nur rudimentär formalisieren lassen“ (VELLUSIG 2011: 159). Dies wirkte sich auch auf die Theorie, d.h. in den Briefstellern, aus. Während ältere Briefsteller, etwa der von JUNCKER (1728), stark regelorientiert und formalisiert waren und bei dem Ausarbeiten von Briefen auf kleinschrittige deduktive Ableitungen unter Anwendung logischer und rhetorischer Schemata rekurrierten, plädierte insbesondere Christian Fürchtegott GELLERT in seiner einflussreichen Praktischen Abhandlung von dem guten Geschmacke in Briefen (1751) für einen ,natürlichen“ und ungezwungenen Sprachgebrauch, der „eine freye Nachahmung des guten Gesprächs“ sein solle und sich deshalb „der Art zu denken und zu reden, die in Gesprächen herrscht mehr nähern [muss], als einer sorgfältigen und geputzten Schreibart“ (S. 3). GELLERTs Ansatz mündet in die Empfehlung:

„Man vergesse also die gewöhnlichen Künste der Briefsteller, wenn man natürliche Briefe schreiben will. Man bekümmere sich dafür um gute Briefe, man lese sie mit Aufmerksamkeit, mehr als einmal, und mache sich mit ihren Tugenden bekannt.“ (1751: 66)

Damit war der Bruch mit der traditionellen Regel-Brieflehre vollzogen. Dies bedeutete freilich nicht, dass es keine weiteren Briefsteller mehr gegeben hätte. (Tatsächlich nahm deren Produktion im deutschen Sprachraum nach 1750 sogar zu. ${ }^{55}$ ). Es kommt jedoch zu einem Funktionswandel: Galt das pedantische Einhalten etablierter Formen bis zur Mitte des 18. Jahrhunderts als standesgemäßes Statussymbol, wird dieses Kopieren nun als Ausdruck von Unselbständigkeit und Ungebildetheit empfunden.

Dieser Einstellungswandel in der Briefkultur wirkte sich auch in anderen Bereichen aus, insbesondere in der Literatur. (Es war kein Zufall, dass eines der erfolgreichsten Werke dieser Zeit, GOETHEs ,Werther', ein Briefroman war. ${ }^{56}$ ) Damit verlor das formale, ,repräsentative' Schreiben an Ansehen. Es hielt sich jedoch - bis zu einem gewissen Grade - in bestimmten Kontexten, und zwar gerade im juristisch-administrativen Bereich. Hiermit einher geht allerdings eine Kritik an diesem Stil, die insbesondere aus

52 Vgl. ZYMNER (2007: Sp. 715), BREMER (2008: 137).

53 Vgl. etwa SCHNEIDERS (Hg.) (1995: 69f.).

54 Zur Rolle der Frauen vgl. auch NICKISCH (1976).

55 Vgl. NICKISCH (1991: 82).

56 Vgl. etwa auch VELLUSIG (2011: 170f.). 
normativ-kritischer Sicht vorgetragen wird und bis in unsere Gegenwart tradiert wird. Beispielsweise hat sich Gustav WUSTMANN (1891) gegen die ,Schreibsprache“ bzw. ,Papiersprache“ gewandt und die Kanzleisprache als ihre besondere Ausprägung kritisiert. Unter ,Schreibsprache“ versteht WUSTMANN ,jene wunderliche schriftliche Ausdrucksweise, die nie gesprochen, sondern immer nur geschrieben wird, und die ausschließlich eine Eigentümlichkeit der deutschen Sprache ist“ (S. 5). Und es gelte:

„Unsere Sprache schleppt [...] aus alter Zeit noch die Fesseln einer unnatürlichen Schreibsprache mit sich [...], dazu die Reste einer umständlichen, wortreichen, gespreizten Kanzleisprache." (S. 5)

„Was man Kanzleisprache, Amtsstil, Aktendeutsch nennt, ist nicht schlechthin dasselbe, wie jene Papiersprache. Alles, was der Papiersprache angehört, gehört natürlich aus dem Amtsstil an, das ist wohl selbstverständlich; aber der Amtsstil ist mehr als die Papiersprache, er hat daneben noch seine besondern Eigentümlichkeiten, er liebt es, sich in die Brust zu werfen, sich breit und wichtig auszudrücken, er hat ein erstaunliches Geschick, die einfachsten Gedanken unter einem unverständlichen oder schwerverständlichen Wortschwall zu vergraben, er hat auch sein besondres Wörterbuch.“ (S. 6)

Ähnlich wird dieser Kanzleistil auch von Ludwig REINERS (1961) kritisiert:

„Der Papierdeutsche glaubt, für seine Verordnungen, Untersuchungen oder Erzählungen sei die Redesprache nicht gut genug. Aber er versucht nicht etwa, sich klarer und zugespitzter auszudrücken, sondern er beschränkt sich darauf, jeden Satzteil durch breitspurige übergenaue Ausdrücke mechanisch zu verlängern. So bevorzugt er bei der Wortwahl lange, zusammengesetzte Wörter, bei der Wortbiegung die Leideform, bei der Wortfügung Gebilde mit umständlichen Verhältniswörtern (Präpositionen) und beim Satzbau die Bandwurm-, Schachtel- und Klemmkonstruktionen.“ (S. 196)

„Als Urheimat des Papierstils gilt das Amtsdeutsch. Wer von Amts wegen Anordnungen oder Urkunden abfaßt, ist verpflichtet, genau zu sein und vorgeschriebene Formen zu gebrauchen. So gerät er leicht in die breitspurige Übergenauigkeit des Papierstils.“ (S. 202)

Differenzierter urteilt der Stilkritiker Eduard ENGEL (1931: 495): „Über den Kanzleistil herrscht Eintracht; er ist nicht schön. Auch ich finde ihn nicht schön“. Jedoch habe dieser Stil „eine gute Eigenschaft“, und zwar „seine Wahrheitsliebe, und die macht sehr viele Untugenden wett“. Zu seinen Untugenden gehöre die „Wichtigtuerei“. Der Kanzleistil

„sagt die Dinge - zwar nicht wie sie sind, aber genau so, wie die Behörde sie aufgefasst wissen will; sagt sie recht ausführlich, oft so sehr, daß der dünne Kern sich in Wortdunst löst; sagt sie im Stil einer papiernen Behörde, eines nebelhaften Begriffes, nicht eines fühlenden Menschen. Aber immerhin, die meisten Kanzleischriften kann man verstehen, was von vielen wissenschaftlichen Schriften nicht gesagt werden kann.“ (S. 495)

In neuerer Zeit gelangt auch Tonio WALTER in seiner Stilkunde für Juristen zu einer 
differenzierteren Einschätzung der Kanzleisprache (2017: 215-221). WALTER setzt das ,Papierdeutsch“ mit dem ,Kanzleideutsch“ gleich und bestimmt als dessen „Urheber“ vornehmlich „die Juristen“ (S. 216). Im Einklang mit der gängigen sprachkritischen Sicht assoziiert er die folgenden Erscheinungen mit dieser Sprachvarietät (S. 216f.): „ein verlängertes Prädikat“ (z.B. Anordnung treffen), ${ }^{57}$ „eine Vorliebe für den Genitiv“, ,verlängerte Präpositionen' (z.B. in Ermangelung statt ohne), den häufigen Gebrauch von Passivkonstruktionen sowie Substantivierungen u.a. In seiner Einschätzung des Kanzleideutsch grenzt WALTER sich hingegen deutlich von der gängigen Auffassung der normativen Stilistik ab:

„Erreicht das Papierdeutsche ein gewisses Ausmaß, wird es ungenießbar. [...] Unterhalb dieser Schwelle mögen viele, zumal Juristen, das Papierdeutsche ganz gern, wenn auch nicht in allen seinen Ausprägungen. Ganz anders die Stilisten. Für sie hört der Spaß beim Papierdeutsch in jedem Falle auf. Sie werden dort regelrecht humorlos und befehden diesen Stil, wo sie ihn treffen, in jeder Erscheinungsform, unerbittlich." (S. 219)

WALTER wendet sich folglich gegen die pauschale Verdammung dieses Sprachgebrauchs durch die ,Stilisten'; eine reflektierte und nicht übertriebene Anwendung sei durchaus zu billigen (S. 218). Hierbei sei insbesondere auf die Wirkung des ,Papierdeutschen' zu achten:

„Sie ist die eines Vortragenden, der die Brauen hochzieht, am Papier zupft, sich räuspert, den Mund spitzt und anhebt, eine gebildete Sprache spazierenzuführen. Das hat ganz gewiss hie und da seine Eleganz und ist dem Leser angenehm. Andererseits ist vor einer Übertreibung zu warnen." (S. 221)

Zudem bemerkt WALTER, dass „ein Gutteil der besten Prosa [...] ihren Charme kanzleideutschen Wendungen“ verdanke (S. 218). Bereits GAUGER (1995: 89) hat angemerkt, dass ein hervorstechender Zug von Franz KAFKAs Prosa „die literarische Anverwandlung des Verwaltungsdeutsch“ sei, und WALTER pflichtet dem bei (S. 218f.). Aber auch bei Thomas MANN findet sich nach WALTER (S. 219) ein ästhetischer Gebrauch der Kanzleisprache, etwa in den Buddenbrooks.

Man kann somit konstatieren, dass die von den Juristen ganz wesentlich mitgeprägte ,Papiersprache' eine mitunter durchaus problematische Varietät darstellt, aber auch interessante stilistische und pragmatische Qualitäten besitzt, die sogar für ästhetische Zwecke ausgenutzt werden können.

57 Somit sind hier also v.a. die viel diskutierten Funktionsverbgefüge gemeint; vgl. hierzu etwa VAN POTTELBERGE (2001). 


\section{Schluss}

Sprachkulturelle Reflexionen haben im juristischen Kontext eine lange Tradition. Dies gilt auch für die deutschsprachige Kultur. Diese Sprachreflexionen der Juristen waren primär durch fachinterne Erfordernisse motiviert: Sie dienten dazu, die Präzision und Verlässlichkeit im juristischen Schrifttum zu erhöhen. Eine solche Disziplinierung des juristischen Schriftsprachgebrauchs wurde schon in den frühneuzeitlichen Formularbüchern angestrebt, und von hier lässt sich eine fachinterne Entwicklungslinie etwa über PÜTTERs Sprachlehre (1767) bis hin zu WALTERs Stilistik (2017) ziehen. Immer wieder haben derartige Werke aber auch über ihren eigentlichen Adressatenkreis hinaus gewirkt und wurden als Impulse für die Weiterentwicklung der deutschen Sprachkultur verstanden. Und Autoren wie RIEDERER, SCHOTTEL, STIELER, PÖLITZ oder SCHMITTHENNER wollten ihre sprachreflektiven Darstellungen auch zweifellos als allgemeinere Beiträge verstanden wissen.

Auch heute können die fachspezifischen sprachpflegerischen Darstellungen der Juristen prinzipiell eine umfassendere Wirkung entfalten. Wenn sich beispielsweise WALTER (2017: 231-244) kritisch mit dem ,Genderdeutsch` auseinandersetzt, behandelt er eine Frage, die für den Gebrauch des Gegenwartsdeutschen von generellem Interesse ist. ${ }^{58}$

Die Geschichte des Einflusses der Juristen auf die deutsche Sprachkultur ist bislang noch nicht in ausführlicherer Weise aufgearbeitet worden. Die hier genannten Beispiele lassen vermuten, dass eine solche Aufgabe lohnend sein könnte.

\section{Literatur}

ALBERTUS, Laurentius (1573): Teutsch Grammatick oder Sprach-Kunst. Augsburg.

ANONYMUS (1744): „CXXXVI. Stück. Einrichtung der Collegien eines studiosi iuris auf der Hallischen Universität“. In: Gelehrte Anzeigen, In alle Wissenschaften... Anderer Theil. Halle: Schneider, S. 734-745.

ASMUTH, Bernhard (1992): „Angemessenheit“. In: UEDING (Hg.) (1992-2012), Bd. 1, S. 579604.

BAHNER, Werner \& Werner NEUMANN (Hgg.) (1985): Sprachwissenschaftliche Germanistik. Ihre Herausbildung und Begründung. Berlin: Akademie-Verlag.

BARNER, Wilfried (1984): „Christian Weise“. In: STEINHAGEN, Harald \& Benno VON WIESE (Hgg.) (1984) Deutsche Dichter des 17. Jahrhunderts. Berlin: Schmidt, S. 690-725.

BENTZINGER, Rudolf (2000): „Die Kanzleisprachen“. In: BESCH, Werner et al. (Hgg.) (2000) Sprachgeschichte. 2. Aufl. Bd. 2. Berlin/New York: de Gruyter, S. 1665-1673.

BOHSE, August [d.i. TALANDER] (1708): Neuvermehrte gründliche Einleitung zur teutschen Oratoria... Jena: E.C. Baillias.

BREMER, Kai (2008): Literatur der Frühen Neuzeit. Paderborn: Fink.

BRITZELMAYR, Wilhelm (1947): „Über die älteste formale Logik in deutscher Sprache“. In: Zeitschrift für philosophische Forschung 2/1947, S. 46-68.

58 Zur feministischen Linguistik und zum ,genderbewussten` Sprachgebrauch vgl. etwa auch RINAS (2011: 132-145). 
CHAUNU, Pierre (1968): Europäische Kultur im Zeitalter des Barock. München/Zürich: Knaur.

CLAJUS, Johannes (1578): Grammatica Germanicae Linguae. Leipzig.

DINZELBACHER, Peter (Hg.) (2008): Europäische Mentalitätsgeschichte. 2. Aufl. Stuttgart: Kröner.

DRESSEL, Gert (1996): Historische Anthropologie. Wien/Köln/Weimar: Böhlau.

VAN DÜLMEN, Richard (1981): „Formierung der europäischen Gesellschaft in der Frühen Neuzeit. Ein Versuch“. In: Geschichte und Gesellschaft 7/1981, S. 20-41.

EGGERS, Hans (1986): Deutsche Sprachgeschichte. Bd. 2. Reinbek: Rowohlt.

ELSTER, Alexander (1901): Methodischer Leitfaden der Deutschen Interpunktionslehre. Magdeburg: Creutz.

ELSTER, Alexander (1906): „Interpunktion“. In: REIN, Wilhelm (Hg.) (1906) Encyklopädisches Handbuch der Pädagogik. 2. Aufl. Bd. 4. Langensalza: Beyer, S. 587-592.

ENGEL, Eduard (1931): Deutsche Stilkunst. 31. Aufl. Leipzig/Wien: Freytag.

ERLER, Julius (1896): Die Sprache des neuen Bürgerlichen Gesetzbuches. Berlin: Verlag des allgemeinen deutschen Sprachvereins.

FIX, Ulla, Andreas GARDT \& Joachim KNAPE (Hgg.) (2008/2009): Rhetorik und Stilistik. 2 Bde. Berlin, New York: de Gruyter.

FRANK, Horst Joachim (1976): Dichtung, Sprache, Menschenbildung. Geschichte des Deutschunterrichts von den Anfängen bis 1945. 2 Bde. München: dtv.

FUCHSBERGER, Ortolf (1533): Ain gründlicher klarer anfang der natürlichen vnd rechten kunst der waren Dialectica. Augspurg (VD16 F 3267).

GARDT, Andreas (1999): Geschichte der Sprachwissenschaft in Deutschland. Berlin, New York: de Gruyter.

GAUGER, Hans-Martin (1995): Über Sprache und Stil. München: Beck.

GELLERT, Christian Fürchtegott (1751): Briefe, nebst einer Praktischen Abhandlung von dem guten Geschmacke in Briefen. Leipzig: Wendler.

GIESECKE, Michael (1992): „,Volkssprache“ und ,Verschriftlichung des Lebens‘ in der frühen Neuzeit.“ In: ders.: Sinnenwandel, Sprachwandel, Kulturwandel. Frankfurt/M.: Suhrkamp, S. 73-121.

GLÜCK, Helmut (2013): Die Fremdsprache Deutsch im Zeitalter der Aufklärung, der Klassik und der Romantik. Wiesbaden: Harrassowitz.

GÖTTERT, Karl-Heinz (2009): Einführung in die Rhetorik. 4. Aufl. Paderborn: Fink.

GÖTZ, Ursula (1992): Die Anfänge der Grammatikschreibung des Deutschen in Formularbüchern des frühen 16. Jahrhunderts. Heidelberg: Winter.

GREULE, Albrecht, Jörg MEIER \& Arne ZIEGLER (Hgg.) (2012): Kanzleisprachenfoschung. Berlin/ Boston: de Gruyter.

HAMMERSTEIN, Notker (Hg.) (1996): Handbuch der deutschen Bildungsgeschichte. Bd. I. München: Beck. HARSDÖRFFER, Georg Philipp (1647): Poetischer Trichter... Nürnberg: Endter.

HERGENHAHN, Richard (1995): „Jakob Köbel zu Oppenheim“. In: Oppenheimer Heft 11, Dezember 1995, S. 3-9.

HINRICHS, Ernst (1980): Einführung in die Geschichte der Frühen Neuzeit. München: Beck.

HUNOLD, Christian Friedrich [MENANTES] (1708): Die Allerneueste Art Höflich und Galant zu Schreiben/ Oder Auserlesene Briefe... Hamburg: Liebernickel.

IGGERS, Wilma Abeles (1967): Karl Kraus. The Hague: Nijhoff.

IM-HOF, Johannes Hieronimus (1687): Send=Schreiben/An Einen Vornehmen Freund/ Uber Einen gehaltenen Discurs... Betreffend die Doctrinam Notarum Distinctionum, E् Interpunctionum... Sultzbach: Lichtenthaler.

JEANNENEY, Jean Noël (2004): Le duel: une passion française, 1789-1914. Paris : Seuil. 
JELLINEK, Max Hermann (1913)/(1914): Geschichte der neuhochdeutschen Grammatik von den Anfängen bis auf Adelung. 2 Bde. Heidelberg: Winter.

JUNCKER, Christian (1728): Der wohl=informirte Brieffsteller... Sechste Auflage. Leipzig: Braun.

KESSEL, Martina (2008): „Individuum/Familie/Geschellschaft: Neuzeit“. In: DINZELBACHER (Hg.) (2008), S. 44-60.

KIENAST, Burkhard (1969): „Keilschrift und Keilschriftliteratur“. In: Frühe Schriftzeugnisse der Menschheit. Göttingen: Vandenhoeck \& Ruprecht, S. 39-55.

KLIX, Friedhart (1993): Erwachendes Denken. Heidelberg/Berlin/Oxford: Spektrum.

KNAPE, Joachim (2000): Allgemeine Rhetorik. Stuttgart: Reclam.

KNAPE, Joachim \& Stefanie LUPPOLD (2008): „Rhetorische und stilistische Praxis des Deutschen in den deutschsprachigen Ländern in Humanismus, Renaissance und Reformation“. In: FIX/ GARDT/KNAPE (Hgg.) (2008/2009), S. 385-413.

KNAPE, Joachim \& Stefanie LUPPOLD (2010): Kommentar zu Friedrich Riederers Spiegel der wahren Rhetorik. Wiesbaden: Harrassowitz.

KRAUS, Karl (1937): Die Sprache. Wien: Verlag ,Die Fackel“.

KRAWIETZ, Werner (1996): „Sprachphilosophie in der Jurisprudenz“. In: DASCAL, Marcelo et al. (Hgg.) (1996) Sprachphilosophie. Bd. 2. Berlin: de Gruyter, S. 1470-1489.

KÜHN, Joachim (1975): Gescheiterte Sprachkritik: Fritz Mauthners Leben und Werk. Berlin, New York: de Gruyter.

LAU, Thomas (2010): Teutschland. Eine Spurensuche 1500 bis 1650. Darmstadt: WBG.

LERCH, Kent D. \& Ralph CHRISTENSEN (2016): „Justiz“. In: JÄGER, Ludwig et al. (Hgg.) (2016) Sprache - Kultur - Kommunikation. Berlin/Boston: de Gruyter Mouton, S. 532-549.

MAUTHNER, Fritz (1923): Beiträge zu einer Kritik der Sprache. 3 Bde. 3. Aufl. Leipzig: Meiner.

MERKEL, Reinhard (1998): Strafrecht und Satire im Werk von Karl Kraus. Frankfurt/M.: Suhrkamp.

MOSER, Fri[e]derich Carl (1749): Versuch Einer Staats-Grammatic... Frankfurt/M.: Andreck.

MOULIN-FANKHÄNEL, Claudine (1994): Bibliographie der deutschen Grammatiken und Orthographielehren. Bd. I. Heidelberg: Winter.

NERIUS, Dieter et al. (2007): Deutsche Orthographie. 4. Aufl. Hildesheim/Zürich/New York: Olms.

NICKISCH, Reinhard M.G. (1969): Die Stilprinzipien in den deutschen Briefstellern des 17. und 18. Jahrhunderts. Göttingen: Vandenhoeck \& Ruprecht.

NICKISCH, Reinhard M.G. (1976): „Die Frau als Briefschreiberin im Zeitalter der deutschen Aufklärung“. In: Wolfenbütteler Studien zur Aufklärung. Bd. III/1976, S. 29-65.

NICKISCH, Reinhard M.G. (1991): Brief. Stuttgart: Metzler.

ÖLINGER, Albert (1573): Vnderricht der Hoch Teutschen Spraach: Grammatica. Straßburg.

OPITZ, Martin (1624): Buch von der Deutschen Poeterey. Brieg/Breslau: Gründer/Müller.

VON POLENZ, Peter (1994): Deutsche Sprachgeschichte vom Spätmittelalter bis zur Gegenwart. Bd. 2. Berlin/New York: de Gruyter.

VON POLENZ, Peter (2000): „Die Sprachgesellschaften und die Entstehung eines literarischen Standards in Deutschland“. In: AUROUX, Sylvain et al. (Hgg.) (2000) Geschichte der Sprachwissenschaften. Bd. 1. Berlin/New York: de Gruyter, S. 827-841.

PÖLITZ, Karl Heinrich Ludwig (1801): Kurze Theorie der Interpunktion nach logischen Grundsätzen. Leipzig: Linke.

PÖLITZ, Karl Heinrich Ludwig (1804): Allgemeine teutsche Sprachkunde, logisch und ästhetisch begründet. Leipzig: Schwickert.

PÖLITZ, Karl Heinrich Ludwig (1805): Systematische Encyklopädie der stylistischen Wissenschaften. Leipzig: Hinrichs. 
VAN POTTELBERGE, Jeroen (2001): Verbonominale Konstruktionen, Funktionsverbgefüge. Heidelberg: Winter.

PUBLICIUS, Jacobus (1482): Artis oratoriae epitoma. Venedig: Ratdolt.

PÜTTER, Johann Stephan (1767): Johann Stephan Pütters ... Zugaben zu seiner Anleitung zur Juristischen Praxi als deren zweyter Theil insonderheit von der Orthographie und Richtigkeit der Sprache... Zweyte Auflage. Göttingen: Vandenhoeck.

RAIBLE, Wolfgang (1991): Die Semiotik der Textgestalt. Heidelberg: Winter.

REINERS, Ludwig (1961): Stilkunst. Ein Lehrbuch deutscher Prosa. Sonderausgabe. München: Beck. RIEDERER, Friedrich (1493 [2009]): Spiegel der wahren Rhetorik. Hg. v. J. KNAPE \& S. LUPPOLD. Wiesbaden: Harrassowitz.

RINAS, Karsten (2011): Sprache, Stil und starke Sprüche. Bastian Sick und seine Kritiker. Darmstadt: Lambert Schneider/WBG.

RINAS, Karsten (2017): Theorie der Punkte und Striche. Die Geschichte der deutschen Interpunktionslehre. Heidelberg: Winter.

ROCKINGER, Ludwig (1863): Briefsteller und formelbücher des eilften bis vierzehnten jahrhunderts. Erste abtheilung. München: Frank.

SCHIROKAUER, Arno (1952): „Frühneuhochdeutsch“. In: STAMMLER, Wolfgang (Hg.) Deutsche Philologie im Aufriss. Band 1. Berlin: Schmidt, Sp. 1014-1076.

SCHMIDT-WIEGAND, Ruth (1987): Jacob Grimm und das genetische Prinzip in Rechtswissenschaft und Philologie. Marburg: Hitzeroth.

SCHMIDT-WIEGAND, Ruth (1998): „Deutsche Sprachgeschichte und Rechtsgeschichte seit dem Ausgang des Mittelalters“. In: BESCH et al. (Hgg.) (1998-2004), S. 87-98.

SCHMITTHENNER, Friedrich (1824): Die Lehre von der Satzzeichnung oder Interpunction in der teutschen Sprache. Frankfurt: Hermann.

SCHMITTHENNER, Friedrich (1828): Teutonia. Aufrührliche Teutsche Sprachlehre. 2 Bde. Frankfurt/M.: Hermann.

SCHOTTEL, Justus Georg (1663): Ausführliche Arbeit von der Teutschen HaubtSprache. Braunschweig: Christoff Friederich Zilliger.

SCHWIND, Peter (1977): Schwulst-Stil. Historische Grundlagen von Produktion und Rezeption manieristischer Sprachformen in Deutschland 1624-1738. Bonn: Bouvier.

SCHWITALLA, Johannes (2002): „Komplexe Kanzleisyntax als sozialer Stil. In: KEIM, Inken \& Wilfried SCHÜTTE (Hgg.) Soziale Welten und kommunikative Stile. Tübingen: Narr, S. 379-398.

STIELER, Kaspar von (1691): Der teutschen Sprache Stammbaum und Fortwachs... [Mit Anhang: Kurze Lehrschrift Von der Hochteutschen Sprachkunst.] Altdorf: Heinrich Meyer.

STIELER, Kaspar von (1705): Deutsche Sekretariat-Kunst... Frankfurt/Leipzig: Andreä.

STÖRIG, Hans Joachim (1988): Kleine Weltgeschichte der Philosophie. 13. Aufl. Frankfurt/M.: Fischer.

THE FAMILY MAGAZINE. Volume IV 1837. London: Ward.

UEDING, Gert (Hg.) (1992-2012): Historisches Wörterbuch der Rhetorik. 10 Bde. Tübingen: Niemeyer / Berlin: de Gruyter.

VELLUSIG, Robert (2000): Schriftliche Gespräche. Briefkultur im 18. Jahrhundert. Wien/Köln/Weimar: Böhlau.

VELLUSIG, Robert (2011): „Aufklärung und Briefkultur.“ In: Das 18. Jahrhundert 35 (2011), Heft 2, S. 154-171.

WALTER, Tonio (2017): Kleine Stilkunde für Juristen. 3. Aufl. München: Beck.

WEISE, Christian (1691): Curiöse Gedancken Von Deutschen Brieffen... Dreßden: Mieth. 
WEITZEL, Jürgen (1994): „Schriftlichkeit und Recht“. In: GÜNTHER, Hartmut \& Otto LUDWIG (Hgg.) (1994) Schrift und Schriftlichkeit. Bd. 1. Berlin/New York: de Gruyter, S. 610-619.

WENDLAND, Ulrich (1930): Die Theoretiker und Theorien der sogen. galanten Stilepoche und die deutsche Sprache. Leipzig: Hermann Eichblatt.

VON WOLZOGEN, Hans (1890): Ueber Verrottung und Errettung der deutschen Sprache. 3. Aufl. Leipzig: Feodor Reinboth.

WUSTMANN, Gustav (1891): Allerhand Sprachdummheiten. Leipzig: Grunow.

WUSTMANN, Gustav (1903): Allerhand Sprachdummheiten. 3. Auflage. Leipzig: Grunow.

ZYMNER, Rüdiger (2007): „Schwulst“. In: UEDING (Hg.) (1992-2012), Bd. 8, S. 706-718.

Doc. Dr. phil. PhDr. Karsten Rinas / K.Rinas@seznam.cz

Univerzita Palackého v Olomouci, Filozofická fakulta, Katedra germanistiky

Krrížkovského 10, 77180 Olomouc, CZ 